\title{
Three Brick Method of the Partial Fraction Decomposition of Some Type of Rational Expression
}

\author{
Damian Słota and Roman Wituła \\ Institute of Mathematics, Silesian University of Technology, Kaszubska 23, \\ 44-100 Gliwice, Poland \\ $\{$ d.slota, rwitula\}@polsl.pl
}

\begin{abstract}
The purpose of this paper is to present a new method of the partial fraction decomposition, the so called "three brick method". The method is of algebraic nature and it is based on simple reduction tasks. The discussed algorithm has been successfully implemented in Mathematica software package.
\end{abstract}

\section{Introduction}

The partial fraction decomposition is a very important topic in symbolic and computational mathematics, for example in inverse Laplace transform. "The residuum method" which is commonly applied to find the inverse Laplace transform, although very legible from the theoretical point of view, turns out as very tedious from the computational point of view (especially if the calculations are finally meant to get rid of the complex notation). The latter often tends to be "the bottleneck" in the whole process of calculating the inverse Laplace transform. Thus, an alternative method of the decomposition of rational functions into partial fractions has evolved considerable interest [2].

In the paper a new method of the partial fraction decomposition, called here "three brick method", is discussed. It is a simple and very efficient method of the decomposition of rational functions:

$$
W(s)=\frac{a s+b}{\left(s^{2}+\alpha s+\beta\right)\left(s^{2}+\gamma s+\delta\right)}
$$

into partial fractions. It is a decomposition method of pure algebraic nature and is based on simple reduction tasks.

\section{Three Brick Method}

Let $\alpha, \beta, \gamma, \delta \in \mathbb{C}, \alpha \neq \gamma$. The case of $\alpha=\gamma$ is expressed in formula (14). Let us put:

$$
M_{1}:=s^{2}+\alpha s+\beta, \quad M_{2}:=s^{2}+\gamma s+\delta
$$


and $M=M_{1} M_{2}$. The method of decomposition of rational function $W(s)$ in the form of $(a, b \in \mathbb{C})$ :

$$
\frac{a s+b}{M}=\frac{A s+B}{M_{1}}+\frac{C s+D}{M_{2}}
$$

is described below.

First, the following system of equations $\left(I_{e q}, I I_{e q}, I I I_{e q}\right)$ is created:

$$
\begin{cases}I_{e q}: & \frac{1}{M_{1}}-\frac{1}{M_{2}}=\frac{M_{2}-M_{1}}{M}=\frac{(\gamma-\alpha) s+\delta-\beta}{M}, \\ I I_{e q}: & \frac{s}{M_{1}}-\frac{s}{M_{2}}=\frac{s\left(M_{2}-M_{1}\right)}{M}=\frac{(\gamma-\alpha) s^{2}+(\delta-\beta) s}{M}, \\ I I I_{e q}: & \frac{2}{M_{1}}-\frac{1}{M_{2}}=\frac{2 M_{2}-M_{1}}{M}=\frac{s^{2}+(2 \gamma-\alpha) s+2 \delta-\beta}{M} .\end{cases}
$$

The next step is to eliminate the summands containing $s^{2}$ from the numerators of the fraction at the right side of equations $I I_{e q}$ and $I I I_{e q}$, thus the fourth equation is generated $\left(I V_{e q}:=I I_{e q}-(\gamma-\alpha) I I I_{e q}\right)$ :

$$
\frac{s-2 \gamma+2 \alpha}{M_{1}}-\frac{s-\gamma+\alpha}{M_{2}}=\frac{[\delta-\beta-(\gamma-\alpha)(2 \gamma-\alpha)] s-(\gamma-\alpha)(2 \delta-\beta)}{M} .
$$

The next two steps of the algorithm involve the elimination of the summands containing $s$ and the free term from the numerators of the fractions at the right side of equations $I_{e q}$ and $I V_{e q} ; V_{e q}:=\left(2 \gamma-\alpha-\frac{\delta-\beta}{\gamma-\alpha}\right) I_{e q}+I V_{e q}$ :

$$
\frac{s+\alpha-\frac{\delta-\beta}{\gamma-\alpha}}{M_{1}}-\frac{s+\gamma-\frac{\delta-\beta}{\gamma-\alpha}}{M_{2}}=\frac{\alpha \delta-\beta \gamma-\frac{(\delta-\beta)^{2}}{\gamma-\alpha}}{M}
$$

and $V I_{e q}:=(2 \delta-\beta) I_{e q}+\frac{\delta-\beta}{\gamma-\alpha} I V_{e q}$ :

$$
\frac{\frac{\delta-\beta}{\gamma-\alpha} s+\beta}{M_{1}}-\frac{\frac{\delta-\beta}{\gamma-\alpha} s+\delta}{M_{2}}=\frac{\left(-\alpha \delta+\beta \gamma+\frac{(\delta-\beta)^{2}}{\gamma-\alpha}\right) s}{M} .
$$

Thanks to the next operation: $\frac{1}{k}\left(-a V I_{e q}+b V_{e q}\right)$, where

$$
k:=\left|\begin{array}{cc}
\alpha & \beta \\
\gamma & \delta
\end{array}\right|-\frac{(\delta-\beta)^{2}}{\gamma-\alpha}
$$

the following decomposition is derived:

$$
\begin{array}{r}
\frac{a s+b}{M}=\frac{1}{k}\left(\frac{\left(b-a \frac{\delta-\beta}{\gamma-\alpha}\right) s+b \alpha-a \beta-b \frac{\delta-\beta}{\gamma-\alpha}}{M_{1}}+\right. \\
\left.-\frac{\left(b-a \frac{\delta-\beta}{\gamma-\alpha}\right) s+b \gamma-a \delta-b \frac{\delta-\beta}{\gamma-\alpha}}{M_{2}}\right) .
\end{array}
$$


Remark. The following equivalent equations hold:

$$
k=0 \Leftrightarrow \delta \alpha^{2}-\gamma(\delta+\beta) \alpha+\gamma^{2} \beta+(\delta-\beta)^{2}=0,
$$

concurrently, the discriminant of the last polynomial of the second order, in consideration of variable $\alpha$ (assuming that $\delta \neq 0$ ) has the following form:

$$
\Delta_{\alpha}=\left(\gamma^{2}-4 \delta\right)(\delta-\beta)^{2} .
$$

Accordingly $\Delta_{\alpha}<0$, if, and only if, the discriminant of the trinomial $M_{2}$ is negative and if $\beta \neq \delta$. The case of $\beta=\delta$ is expressed in formulas:

$$
\begin{aligned}
& \frac{1}{\left(s^{2}+\alpha s+\beta\right)\left(s^{2}+\gamma s+\beta\right)}=\frac{1}{\beta(\alpha-\gamma)}\left(\frac{s+\alpha}{s^{2}+\alpha s+\beta}-\frac{s+\gamma}{s^{2}+\gamma s+\beta}\right), \\
& \frac{s}{\left(s^{2}+\alpha s+\beta\right)\left(s^{2}+\gamma s+\beta\right)}=\frac{1}{\gamma-\alpha}\left(\frac{1}{s^{2}+\alpha s+\beta}-\frac{1}{s^{2}+\gamma s+\beta}\right) .
\end{aligned}
$$

If $\alpha=\gamma$ (assuming that $M_{1} \neq M_{2}$ ) the following decomposition is derived:

$$
\frac{a s+b}{\left(s^{2}+\alpha s+\beta\right)\left(s^{2}+\alpha s+\delta\right)}=\frac{1}{\delta-\beta}\left(\frac{a s+b}{s^{2}+\alpha s+\beta}-\frac{a s+b}{s^{2}+\alpha s+\delta}\right) .
$$

\section{$3 \quad$ Algorithm in Mathematica}

The algorithm discussed in the previous section will next be implemented in Mathematica [1,3]. The summands connected with trinomials $M_{1}$ and $M_{2}$ shall be expressed in separate notations, so that the transformations are performed separately on both fractions. We start with defining the trinomials:

In [1]: $=\mathrm{m} 1=\mathrm{s}^{2}+\alpha \mathrm{s}+\beta ; \mathrm{m} 2=\mathrm{s}^{2}+\gamma \mathrm{s}+\delta ;$

Let us form three equations $\left(I_{e q}, I I_{e q}, I I I_{e q}\right)$ :

$$
\begin{aligned}
\text { In }[2]:= & \text { eq } 1=\{1 / \mathrm{m} 1,-1 / \mathrm{m} 2\} ; \\
\text { eq } 2 & =\{\mathrm{s} / \mathrm{m} 1,-\mathrm{s} / \mathrm{m} 2\} ; \\
& \text { eq } 3=\{2 / \mathrm{m} 1,-1 / \mathrm{m} 2\} ;
\end{aligned}
$$

The summands containing $s^{2}$ are eliminated $\left(I V_{e q}\right)$ :

In [3] : = eq $4=$ Simplify [eq $2-(\gamma-\alpha)$ eq3]

Out $[3]=\left\{\frac{\mathrm{s}+2 \alpha-2 \gamma}{\mathrm{s}^{2}+\mathrm{s} \alpha+\beta},-\frac{\mathrm{s}+\alpha-\gamma}{\mathrm{s}^{2}+\mathrm{s} \gamma+\delta}\right\}$

The next summands containing $s$ are eliminated $\left(V_{e q}\right)$ :

$$
\begin{aligned}
& \text { In }[4]:=\text { eq } 5=\operatorname{Simplify}\left[\left(2 \gamma-\alpha-\frac{\delta-\beta}{\gamma-\alpha}\right) \text { eq } 1+\text { eq } 4\right] \\
& \text { Out }[4]=\left\{\frac{\alpha^{2}-\beta+\mathrm{s}(\alpha-\gamma)-\alpha \gamma+\delta}{\left(\mathrm{s}^{2}+\mathrm{s} \alpha+\beta\right)(\alpha-\gamma)}, \frac{\beta-\alpha \gamma+\gamma^{2}+\mathrm{s}(-\alpha+\gamma)-\delta}{(\alpha-\gamma)\left(\mathrm{s}^{2}+\mathrm{s} \gamma+\delta\right)}\right\}
\end{aligned}
$$


The elimination of the free term $\left(V I_{e q}\right)$ :

In $[5]:=$ eq $6=\operatorname{Simplify}\left[(2 \delta-\beta)\right.$ eq $1+\left(\frac{\delta-\beta}{\gamma-\alpha}\right)$ eq 4$]$
Out $[5]=\left\{\frac{\beta(\alpha-\gamma)+\mathrm{s}(\beta-\delta)}{\left(\mathrm{s}^{2}+\mathrm{s} \alpha+\beta\right)(\alpha-\gamma)}, \frac{(-\alpha+\gamma) \delta+\mathrm{s}(-\beta+\delta)}{(\alpha-\gamma)\left(\mathrm{s}^{2}+\mathrm{s} \gamma+\delta\right)}\right\}$

The definition of parameter $k$ :

$\operatorname{In}[6]:=\mathrm{k}=\operatorname{Det}\left[\left(\begin{array}{ll}\alpha & \beta \\ \gamma & \delta\end{array}\right)\right]-\frac{(\delta-\beta)^{2}}{\gamma-\alpha}$

Final decomposition:

$$
\begin{aligned}
\text { In [7] : }= & \text { up }=\operatorname{Total}\left[\frac{1}{\mathrm{k}}(-\mathrm{a} \text { eq } 6+\mathrm{b} \text { eq } 5)\right] \\
\text { Out }[7]= & \frac{-\frac{\mathrm{a}(\beta(\alpha-\gamma)+\mathrm{s}(\beta-\delta))}{\left(\mathrm{s}^{2}+\mathrm{s} \alpha+\beta\right)(\alpha-\gamma)}+\frac{\mathrm{b}\left(\alpha^{2}-\beta+\mathrm{s}(\alpha-\gamma)-\alpha \gamma+\delta\right)}{\left(\mathrm{s}^{2}+\mathrm{s} \alpha+\beta\right)(\alpha-\gamma)}}{-\beta \gamma+\alpha \delta-\frac{(-\beta+\delta)^{2}}{-\alpha+\gamma}}+ \\
& \frac{\frac{\mathrm{b}\left(\beta-\alpha \gamma+\gamma^{2}+\mathrm{s}(-\alpha+\gamma)-\delta\right)}{(\alpha-\gamma)\left(\mathrm{s}^{2}+\mathrm{s} \gamma+\delta\right)}-\frac{\mathrm{a}((-\alpha+\gamma) \delta+\mathrm{s}(-\beta+\delta))}{(\alpha-\gamma)\left(\mathrm{s}^{2}+\mathrm{s} \gamma+\delta\right)}}{-\beta \gamma+\alpha \delta-\frac{(-\beta+\delta)^{2}}{-\alpha+\gamma}}
\end{aligned}
$$

The Apart instruction cannot be used for the derivation of above decomposition:

$$
\begin{aligned}
& \operatorname{In}[8]:=\operatorname{Apart}[(\mathrm{as}+\mathrm{b}) /(\mathrm{m} 1 \mathrm{~m} 2)] \\
& \operatorname{Out}[8]=\frac{\mathrm{b}+\mathrm{a} \mathrm{s}}{\left(\mathrm{s}^{2}+\mathrm{s} \alpha+\beta\right)\left(\mathrm{s}^{2}+\mathrm{s} \gamma+\delta\right)}
\end{aligned}
$$

\section{Conclusion}

In this paper a new method of the partial fraction decomposition, the three brick method, is presented. It is a simple and effective method of an algebraic nature, based on simple reduction procedures. The discussed algorithm has successfully been used in Mathematica software package. The advantage of the method is the possibility to perform computations for symbolic data, which is not always possible by using the standard Mathematica commands (Apart).

\section{References}

1. Drwal, G., Grzymkowski, R., Kapusta, A., Słota, D.: Mathematica 5. WPKJS, Gliwice (2004) (in Polish)

2. Grzymkowski, R., Wituła, R.: Computational Methods in Algebra, part I. WPKJS, Gliwice (2000) (in Polish)

3. Wolfram, S.: The Mathematica Book, 5th ed. Wolfram Media, Champaign (2003) 